\title{
Prospecção Científica e Tecnológica do Gênero Ageratum
}

\author{
Technological and Scientific Prospection of Ageratum Genus
}

\author{
Ingrid Estefania Mancia de Gutiérrez ${ }^{1}$ \\ Daniel da Silva de Jesus ${ }^{1}$ \\ Lenaldo Muniz de Oliveira ${ }^{1}$ \\ Angélica Maria Lucchese ${ }^{1}$ \\ ${ }^{1}$ Universidade Estadual de Feira de Santana, Feira de Santana, BA, Brasil
}

\begin{abstract}
Resumo
Ageratum (Asteraceae) é nativo das Américas, sendo A. conyzoides a espécie mais explorada para diversos fins em diferentes partes do mundo. O objetivo deste trabalho foi prospectar publicações científicas e patentes com os representantes do gênero. A consulta foi realizada em bancos de publicações científicas e de patentes, utilizando os termos Ageratum e mentrasto. Foi possível observar um expressivo número de publicações científicas (761) em detrimento dos depósitos de patentes (294) e em ambos os documentos se destaca a área medicinal e agronômica, sendo a China a maior detentora dos depósitos (90\%). Nenhuma patente concedida ou em avaliação foi encontrada no Instituto Nacional da Propriedade Industrial (INPI), apesar de terem sido compiladas 37 dissertações/teses no Brasil na área farmacológica e agronômica, campos de grande interesse comercial. Isso demonstra a falta de incentivo à propriedade intelectual na Academia do País e a necessidade das pesquisas estarem alinhadas para solucionar alguma carência da sociedade.
\end{abstract}

Palavras-chave: Patentes. Planta medicinal. Mentrasto.

\begin{abstract}
Ageratum (Asteraceae) is native to the Americas and A. conyzoides is the most exploited species for a variety of purposes in different parts of the world. The aim of this study was to investigate scientific publications and patents with representatives of the genus. The survey used the terms Ageratum and goat weed. A significant number of scientific publications (761) compared to patent deposits (294) were observed, with China holding the largest number of deposits (90\%). No patent was awarded or under consideration at INPI (National Institute of Industrial Property), despite the fact that 37 dissertations/theses have been submitted in Brazil, in the area of pharmacology and agronomy, fields of considerable commercial interest. This demonstrates the lack of support for intellectual property in academic settings and the need for research to bridge societal gaps.
\end{abstract}

Keywords: Patents. Medicinal plant. Goat weed.

Área tecnológica: Prospecção científica e tecnológica. 


\section{Introdução}

O gênero Ageratum (Asteraceae) possui cerca de 40 espécies de plantas herbáceas (ENCICLOPEDIA BRITANICA, 2018), tendo quatro representantes identificadas no Brasil (A. candidum G.M.Barroso; A. conyzoides L.; A. fastigiatum (Gardner) R.M.King \& H.Rob. e A. myriadenium (Sch.Bip. ex Baker) R.M.King \& H.Rob.), segundo dados da Lista de Espécies da Flora do Brasil (NAKAJIMA, 2016). Dos representantes do gênero, apenas A. iltisii consta na Lista Vermelha da União Internacional para a Conservação da Natureza (IUCN Red List), classificada como espécie vulnerável, e nenhuma espécie do gênero foi encontrada na Lista Vermelha da Flora do Brasil (MARTINELLI; MORAES, 2013).

Dentre as espécies do gênero, $A$. conyzoides é a mais estudada devido ao seu potencial medicinal (DEL-VECHIO-VIEIRA et al., 2008) e no Brasil é popularmente conhecida como mentrasto ou erva-de-são-joão (BOSI et al., 2013). A. conyzoides fez parte do elenco de 74 espécies vegetais selecionadas pelo Programa de Pesquisas de Plantas Medicinais da extinta Central do Medicamento (CEME), sendo confirmados seus benefícios para o tratamento da artrose, sem atribuição de toxicidade, por meio de estudos de farmacologia e toxicologia pré-clínica e clínica (BRASIL, 2006). Há o registro de fitoterápicos manipulados de A. conyzoides nas Farmácias Vivas (FV) do País, a exemplo das FV de Fortaleza (SILVA et al., 2006) e do Distrito Federal (COSTA et al., 1992), sendo indicados para tratar reumatismo. As doenças inflamatórias, incluindo diferentes tipos de reumatismo, são a maior causa de morbidade decorrente das atividades laborais em todo o mundo, e os fitoterápicos vêm ganhando destaque no tratamento dessas doenças por conta dos efeitos colaterais provocados pelas drogas sintéticas (JAWAID; SHUKLA; VERMA, 2011; GRIFFIN, 2013).

Uma prospecção sistematizada da literatura especializada sobre plantas medicinais aumenta o conhecimento sobre esses vegetais por parte das instituições de pesquisa, empresas e órgãos reguladores, como também guia a realização de novos estudos dirigidos, de modo a complementar informações existentes para espécies medicinais de interesse da população brasileira (CARVALHO, 2011). A busca do estado da técnica apenas em artigos científicos mostra dados defasados ante a real situação do desenvolvimento científico e tecnológico de qualquer área de conhecimento, uma vez que nos países desenvolvidos o depósito de patente antecede a publicação de artigos científicos (BUENO, 2014). O conteúdo dos documentos de patente é fonte formal de informação, por meio da qual se pode ter acesso a dados de invenções que, muitas vezes, não estão disponíveis em livros nem em artigos científicos (SERAFINI et al., 2012). Assim, a partir da prospecção tecnológica é possível definir as áreas de pesquisa em que os estudos são escassos e traçar estratégias para solucionar determinado problema no mercado (BARROS, 2014), em especial o farmacêutico, influenciando assim de forma significativa a sociedade como um todo.

Nesse sentido, este trabalho teve como objetivo realizar o levantamento de patentes, além de artigos, teses e dissertações relacionadas ao gênero Ageratum, averiguando quais as espécies e em quais áreas do conhecimento estão sendo mais investigadas, e assim nortear a realização de novos estudos, e também verificar a correlação existente entre o quantitativo de depósitos de patentes e a produção científica. 


\section{Metodologia}

A estratégia de busca realizada foi definida utilizando-se o termo Ageratum nas bases de dados Scientific Electronic Library Online (SciELO), Science Direct, Lilacs, Medline (via PubMed), Centro Latino-Americano e do Caribe de Informação em Ciências da Saúde (BIREME), Scopus e SciFinder, para o período anterior a fevereiro de 2015. As referências e os resumos dos trabalhos obtidos a partir da consulta a essas bases de dados foram exportados para o programa EndNote Web versão 3.5, utilizando-se a ferramenta disponibilizada pelo programa para a remoção dos trabalhos repetidos. Os artigos científicos foram analisados quanto às categorias do assunto. O banco de teses da Coordenação de Aperfeiçoamento de Pessoal de Nível Superior (Capes) também foi utilizado na busca, que foi realizada em período anterior a agosto de 2013, analisando-se o assunto e o estado brasileiro a qual pertence o programa de pós-graduação.

Com relação às patentes, a busca foi realizada para o período anterior a janeiro de 2015 com os termos "Ageratum" e "mentrasto" no campo de título ou resumo, nos bancos de patentes públicos do Instituto Nacional da Propriedade Industrial (INPI), no banco europeu, European Patent Office, (EPO) e no banco da Organização Mundial de Propriedade Intelectual (OMPI). Os parâmetros de análise foram o ano de depósito, titularidade dos depositantes, países depositantes e Classificação Internacional de Patentes (CIP).

Os documentos encontrados tiveram suas informações transferidas para o software Microsoft Office Excel 2013, no qual foi possível analisar os dados tabelados e expressá-los por meio da frequência encontrada.

\section{Resultados e Discussão}

A prospecção científica permitiu confirmar que, entre os representantes do gênero, A. conyzoides é a mais estudada com 580 (76,2\%) publicações, seguida de 88 (11,6\%) para A. houstonianum (Tabela 1).

Tabela 1 - Número de publicações científicas para o gênero Ageratum para o período anterior a fevereiro de 2015

$\begin{array}{ccc}\text { EsPÉCIE } & \mathbf{N} . & \mathbf{\%} \\ \text { A. conyzoides } & 580 & 76,22 \\ \text { A. houstonianum } & 88 & 11,56 \\ \text { Ageratum sp. } & 72 & 9,46 \\ \text { A. fastigiatum } & 9 & 1,18 \\ \text { A. corymbosum } & 4 & 0,53 \\ \text { A. gaumeri } & 2 & 0,26 \\ \text { A. myriadenia } & 2 & 0,26 \\ \text { A. mexicanum } & 1 & 0,13 \\ \text { A. strictum } & 1 & 0,13 \\ \text { A. riparium } & 1 & 0,13 \\ \text { A. tomentosum } & 1 & 0,13 \\ \text { Total } & \mathbf{7 6 1} & \mathbf{1 0 0}\end{array}$

Fonte: Scientific Electronic Library Online SienceDirect, Lilacs, Medline, Centro Latino-Americano e do Caribe de Informação em Ciências da Saúde, Scopus e SciFinder (2018) 
O maior número de estudos para o gênero concentra-se na área da fitopatologia, 16,4\% (125) (Tabela 2), e refere-se à doença da nervura amarela causada por um complexo de DNA viral de um begomovirus (Geminiviridae) que ocorre em Ageratum.

Os trabalhos de ecologia reunidos (Tabela 2) incluem estudos com agroecologia, banco de germoplasma, fungos endofíticos, flora apícola e biomonitoramento da poluição ambiental. Os estudos de botânica para o gênero abrangem os ramos da palinologia, filogenética, anatomia, fitossociologia e taxonomia. Dos trabalhos classificados como Revisão, 5,8\% (44) (Tabela 2), apenas $9,1 \%$ (4) especificam um representante do gênero, sendo todos de A. conyzoides (OKUNADE, 2002; KONG et al., 2004; KAMBOJ; SALUJA, 2008; KAUR et al., 2012). Das publicações tratadas como Outras, 1,4\% (11) foram reunidas nos temas de ação algicida, ação repelente para uso em humanos, aplicação na indústria têxtil, alérgenos em humanos, FV, biogás e cultivares.

Para A. houstonianum prevalecem os estudos na área de fisiologia vegetal, seguidos dos estudos relacionados ao controle de pragas e para $A$. conzyoides as publicações dominam a área de farmacologia pré-clínica, seguidos da área de etnofarmacologia (Tabela 2). Os ensaios farmacológicos in vivo confirmam a ação analgésica, anti-inflamatória, espasmolítica, gastroprotetora, hepatoprotetora, cicatrizante, hipoglicemiante, antiurolítica, antimalárica, hematopoiética e radioprotetora dos derivados de $A$. conyzoides (dados não apresentados).

A atividade biológica para fins agronômicos também tem sido investigada em $A$. conyzoides. Estudos de alelopatia abrangem 6,6\% (38) dos trabalhos, a ação inseticida no controle de pragas tem sido verificada em 7,1\% (41) dos estudos, enquanto a ação fungicida, bactericida, nematicida contra fitopatógenos são reportadas em 3,1\% (18) das publicações (Tabela 2). No entanto, percebe-se que vários campos de investigação continuam com lacunas abertas para a descoberta de novas propriedades biológicas para as espécies de Ageratum.

Tabela 2 - Distribuição das publicações científicas segundo área de estudo para o gênero Ageratum, para o período anterior a fevereiro de 2015

\begin{tabular}{|c|c|c|c|c|c|c|c|c|c|c|c|c|}
\hline Classificação & 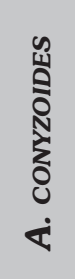 & 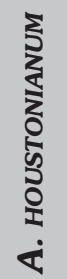 & 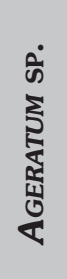 & 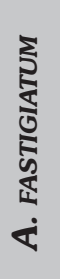 & 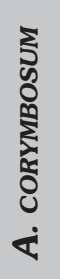 & $\begin{array}{l}\overline{8} \\
\text { w } \\
\text { s. } \\
\text { \& } \\
\dot{\alpha}\end{array}$ & 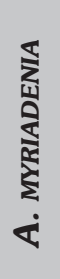 & 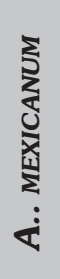 & 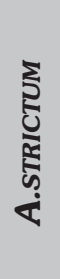 & 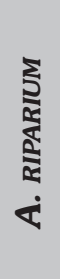 & 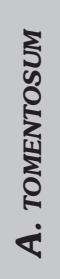 & TOTAL \\
\hline \multicolumn{13}{|c|}{ Abrangendo Apenas uma Área de Estudo } \\
\hline Ação contra fitopatógenos & 13 & 3 & 1 & - & - & 2 & - & - & - & - & - & 19 \\
\hline Alelopatia & 35 & 1 & - & - & - & - & - & - & - & - & - & 36 \\
\hline Ecologia & 61 & 13 & 1 & - & - & - & - & - & - & - & - & 75 \\
\hline Fisiologia vegetal & 13 & 16 & 3 & 1 & 1 & - & - & - & - & - & - & 34 \\
\hline Fitopatologia & 64 & 6 & 55 & - & - & - & - & - & - & - & - & 125 \\
\hline Fitorremediação & 4 & 1 & - & - & - & - & - & - & - & - & - & 5 \\
\hline Controle de pragas & 33 & 14 & - & 2 & - & - & - & - & - & - & - & 49 \\
\hline Manejo de plantas daninhas & 45 & 3 & 1 & - & - & - & - & - & - & - & - & 49 \\
\hline Veterinária & 5 & 2 & - & - & - & - & - & - & - & - & - & 7 \\
\hline $\begin{array}{l}\text { Etnofarmacologia/ } \\
\text { Etnobotânica (E/E) }\end{array}$ & 57 & 4 & - & - & 1 & - & - & - & - & - & - & 62 \\
\hline
\end{tabular}


Botânica

Controle de qualidade (CQ)

$$
1
$$

Química/Fitoquímica (Q/F)

$44 \quad 12$

Farmacologia pré-clínica (FPC)

$75 \quad 1$

65

Farmacologia clínica

Toxicologia pré-clínica (TPC)

Outros

1

Revisão

\section{5}

$8 \quad 1 \quad 2$

11

$\begin{array}{lll}36 & 3 & 4\end{array}$

44

\section{Abrangendo Mais de uma Área de Estudo}

Botânica - Ecologia

Botânica - Manejo de plantas daninhas

Ecologia - Manejo de plantas daninhas

Ecologia - Controle de pragas

Ecologia - Fisiologia vegetal

E/E - Botânica

$\mathrm{E} / \mathrm{E}-$ Ecologia

E/E - Ação contra fitopatógenos

$$
\mathrm{E} / \mathrm{E}-\mathrm{FPC}
$$

$\mathrm{E} / \mathrm{E}-\mathrm{Q} / \mathrm{F}-\mathrm{FPC}$

$$
\mathrm{E} / \mathrm{E}-\mathrm{CQ}
$$

$\mathrm{Q} / \mathrm{F}$ - Ecologia

$\mathrm{Q} / \mathrm{F}$ - Fisiologia vegetal

$$
\text { Q/F - FPC }
$$

Q/F - FPC - Antiaflatoxina

$\mathrm{Q} / \mathrm{F}$ - Antiaflatoxina

$$
\text { Q/F - Alelopatia }
$$

$\mathrm{Q} / \mathrm{F}$ - Controle de pragas

$\mathrm{Q} / \mathrm{F}$ - Ação contra fitopatógenos

Q/F - Manejo de plantas daninhas

$$
\mathrm{Q} / \mathrm{F}-\mathrm{CQ}
$$

$\mathrm{Q} / \mathrm{F}$ - Ensaio in silico

FPC - Ação contra fitopatógenos

$$
\begin{gathered}
\text { FPC - TPC - CQ } \\
\text { FPC - TPC }
\end{gathered}
$$

\section{Total}

1

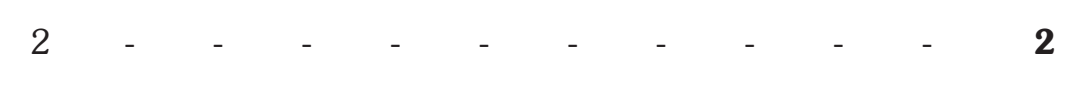

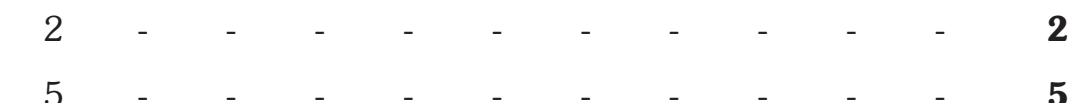

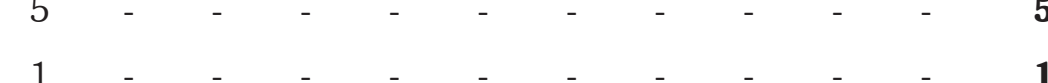

Fonte: SienceDirect, Lilacs, Medline, Centro Latino-Americano e do Caribe de Informação em Ciências da Saúde (BIREME), Scopus e SciFinder (2018) 
No Banco de Teses da Capes, em 2013, constavam 37 dissertações e seis teses defendidas nas instituições de ensino do País, todas com $A$. conyzoides nas diferentes áreas do conhecimento (Quadro 1). Com a atualização da plataforma da Capes, posterior a data da busca, as dissertações/teses anterior a 2011 saíram do endereço eletrônico, no entanto, os títulos dos trabalhos encontram-se aqui compilados (Quadro 1).

Quadro 2 - Dissertações e teses referentes à A. conyzoides (busca em ago. 2013)

\begin{tabular}{|c|c|c|}
\hline Ano & Título DA DISSERTAÇÃO/TESE & $\begin{array}{l}\text { INSTITUIÇÃO } \\
\text { DE ENSINO }\end{array}$ \\
\hline 1991 & $\begin{array}{l}\text { Possível inibidor de fosfodiesterase para AMP cíclico } \\
\text { em folhas de Ageratum conyzoides. }\end{array}$ & UFC \\
\hline 1995 & $\begin{array}{c}\text { Influência de alguns derivados de vegetais da família Compositae } \\
\text { sobre o comportamento de oviposicao, eclosão larvaria e atratividade } \\
\text { para Aedes (ochlerotatus) fluviatilis (LUTZ, 1904). }\end{array}$ & UFMG \\
\hline 1996 & $\begin{array}{l}\text { Produção de biomassa e teor do óleo essencial em função das fases de } \\
\text { desenvolvimento, calagem e adubação mineral e orgânica em Ageratum conyzoides L. }\end{array}$ & UNESP* \\
\hline 1997 & $\begin{array}{l}\text { Efeitos de capinas e adubação nitrogenada em cobertura em diferentes } \\
\text { estádios de desenvolvimento do feijoeiro, em distintas épocas de plantio. }\end{array}$ & UFV \\
\hline 1997 & $\begin{array}{l}\text { Avaliação do efeito antagonista de quatro espécie de plantas medicinais } \\
\text { sobre a população de Meloidogyne incognita [(KOFOID \& WHITE, } \\
\text { 1919) CHITWOOD, 1949] no solo, em estufa plástica. }\end{array}$ & UEM \\
\hline 1998 & $\begin{array}{l}\text { Avaliações de desempenho germinativo de sementes } \\
\text { de mentrasto ("Ageratum conyzoides" L.) }\end{array}$ & UEM \\
\hline 1998 & $\begin{array}{l}\text { Efeito de "Cymbopogon citratus", "Eucalyptus citriodora", } \\
\text { "Ageratum conyzoides " e "Achillea millefolium" no crescimento } \\
\text { vegetativo de "Didymella bryoniae" (Auersw.) Rehm. }\end{array}$ & UEM \\
\hline 1998 & $\begin{array}{l}\text { Purificação, caracterização e estudo toxicológico das substâncias hidrossolúveis } \\
\text { de Ageratum conyzoides com atividades analgésica e antiinflamatória }\end{array}$ & UFC \\
\hline 1999 & $\begin{array}{l}\text { Avaliação do consórcio milho, leguminosas para } \\
\text { adubação verde e plantas espontâneas }\end{array}$ & UFV \\
\hline 2000 & $\begin{array}{l}\text { Alcalóides Pirrolizidínicos em Plantas Brasileiras de Interesse } \\
\text { Alimentar e Medicinal: Identificação e Toxicologia }\end{array}$ & UNICAMP \\
\hline 2000 & $\begin{array}{l}\text { Alternativas terapêuticas na prevenção da cistite hemorrágica induzida } \\
\text { por ciclofosfamida e ifosfamida com drogas antiinflamatórias: } \\
\text { Um estudo experimental comparativo com o mesma }\end{array}$ & UFC \\
\hline 2001 & $\begin{array}{l}\text { Mapeamento da variabilidade espacial das plantas daninhas com } \\
\text { a utilização de ferramentas da agricultura de precisão }\end{array}$ & USP \\
\hline 2001 & $\begin{array}{l}\text { Isolamento, Identificação e Atividade Inseticida de } \\
\text { Constituintes Químicos de Ageratum conyzoides }\end{array}$ & UFV \\
\hline 2002 & $\begin{array}{l}\text { Aspectos etnobotânicos do trabalho com plantas medicinais } \\
\text { realizados por curandeiros no município de Iporanga/SP }\end{array}$ & UNESP** \\
\hline 2002 & $\begin{array}{l}\text { Germinação, fenologia, propagação vegetativa e } \\
\text { diferentes idades de corte do mentrasto }\end{array}$ & UFC \\
\hline 2002 & $\begin{array}{l}\text { Transmissibilidade da leprose nas cercas-vivas, quebra-ventos e plantas daninhas } \\
\text { para citros através de brevipalpus phoenicis (GEIJSKES, 1939) (Acari: Tenuipalpidae) }\end{array}$ & UNESP* \\
\hline 2003 & $\begin{array}{l}\text { Soluções homeopáticas, crescimento e produção de compostos } \\
\text { bioativos em Ageratum conyzoides L. (Asteraceae) }\end{array}$ & UFV \\
\hline
\end{tabular}




\begin{tabular}{|c|c|c|}
\hline Ano & Título DA DisSERTAÇÃO/TESE & $\begin{array}{l}\text { INSTITUIÇÃO } \\
\text { DE ENSINO }\end{array}$ \\
\hline 2003 & $\begin{array}{l}\text { Influência da cultura do algodoeiro e plantas daninhas associadas na biologia do } \\
\text { percevejo predador Podisus nigrispinus (DALLAS, 1851) (Heteroptera:Pentatomidae) }\end{array}$ & UFRPE \\
\hline 2004 & $\begin{array}{l}\text { Efeito de substratos alternativos no controle biológico de } \\
\text { ácaros-praga da seringueira (Hevea brasiliensis) }\end{array}$ & UNESP* \\
\hline 2004 & $\begin{array}{l}\text { Constatação da transmissibilidade do vírus da leprose dos citros por } \\
\text { Brevipalpus phoenicis (GEIJSKES, 1939) em cercas vivas, quebra-ventos } \\
\text { e plantas daninhas através da microscopia eletrônica de transmissão }\end{array}$ & UNESP* \\
\hline 2005 & $\begin{array}{l}\text { Levantamento de vírus em tomateiros na região produtora da Ibiapaba- } \\
\text { CE e identificação de fontes alternativas de begomovirus }\end{array}$ & UFC \\
\hline 2005 & $\begin{array}{l}\text { Girassol (Helianthus annuus L.) resistente às imidazolinonas: } \\
\text { obtenção de genótipo e manejo de plantas daninhas }\end{array}$ & UEL \\
\hline 2005 & $\begin{array}{l}\text { Ensaios in vitro para testar a ação antifúngica de } \\
\text { extratos vegetais em espécies de Aspergillus }\end{array}$ & UFRRJ \\
\hline 2005 & $\begin{array}{l}\text { Efeito de diferentes períodos de convivência com as plantas daninhas } \\
\text { sobre a produtividade da cultura da cenoura (Daucus carota L.) }\end{array}$ & UNESP* \\
\hline 2005 & $\begin{array}{l}\text { Avaliação da atividade antifúngica de extratos de extratos aquosos } \\
\text { de plantas sobre espécies toxígenas de Aspergillus }\end{array}$ & UFRRJ \\
\hline 2006 & $\begin{array}{l}\text { Diversidade de ácaros em cultivo orgânico de citros e na } \\
\text { vegetação natural circundante, e perspectivas para a criação } \\
\text { massal de Iphiseiodes zuluagai (Acari: Phytoseiidae) }\end{array}$ & UNESP* \\
\hline 2007 & Avaliação psicofarmacológica de Ageratum conyzoides L. Asteraceae & UNESC \\
\hline 2007 & $\begin{array}{l}\text { Morfo-anatomia, fitoquímica e atividade antimicrobiana } \\
\text { de Ageratum conyzoides L. Asteracea }\end{array}$ & UFG \\
\hline 2008 & $\begin{array}{l}\text { Avaliação da reação de espécies de plantas daninhas } \\
\text { ao nematoide Meloidogyne paranaenses }\end{array}$ & UEL \\
\hline 2008 & $\begin{array}{l}\text { Atividade inseticida de pós vegetais e do gesso em relação ao caruncho-do- } \\
\text { feijão, Zabrotes subfasciatus (BOHEMAN, 1833) (Coleoptera: Chrysomiledae) }\end{array}$ & UFAL \\
\hline 2008 & $\begin{array}{l}\text { Interferência de palhadas de sorgo, capim braquiária e } \\
\text { milheto sobre a cultura do milho e plantas daninhas }\end{array}$ & UFLA \\
\hline 2008 & $\begin{array}{l}\text { Repelência de extratos de plantas e do deet (n, n-diethyl-m- } \\
\text { toluamide) em Amblyomma cajennense (Acari: Ixodidae) }\end{array}$ & UFG \\
\hline 2009 & $\begin{array}{l}\text { Quimioprevenção pelo óleo essencial de mentrasto (Ageratum conyzoides) } \\
\text { no crescimento de Aspergillus flavus e da produção de aflatoxina }\end{array}$ & $\begin{array}{l}\text { Instituto } \\
\text { Biológico-SP }\end{array}$ \\
\hline 2010 & $\begin{array}{l}\text { Seleção de progênies e estimativas de parametros genéticos em Ageratum } \\
\text { conyzoides L. (Asteraceae) para caracteres morfo-agronômicos }\end{array}$ & UEFS \\
\hline 2010 & $\begin{array}{l}\text { Avaliação da atividade antimicrobiana e triagem fitoquímica dos extratos } \\
\text { de espécies da família Asteraceae encontradas no semi-árido baiano }\end{array}$ & UEFS \\
\hline 2010 & $\begin{array}{c}\text { Produtos naturais e fungos entomopatogênicos para o manejo de Coptotermes } \\
\text { gestroi (Isoptera: Rhinotermitidae) em árvores de área urbana }\end{array}$ & $\begin{array}{l}\text { Instituto } \\
\text { Biológico-SP }\end{array}$ \\
\hline 2011 & $\begin{array}{l}\text { Óleos essenciais de mentrasto e orégano no controle } \\
\text { de Aspergillus flavus em milho e soja }\end{array}$ & $\begin{array}{l}\text { Instituto } \\
\text { Biológico-SP }\end{array}$ \\
\hline 2012 & $\begin{array}{l}\text { Diversidade e bioprospecção de fungos endofíticos associados a } \\
\text { plantas bioativas de diferentes ecossistemas brasileiros }\end{array}$ & UFMG \\
\hline 2012 & Presença de alcaloides pirrolizidínicos em Ageratum conyzoides L., Asteraceae & UFSC \\
\hline
\end{tabular}




\begin{tabular}{|c|c|c|}
\hline ANo & Título DA DISSERTAÇÃo/TESE & $\begin{array}{c}\text { INSTITUIÇÃo } \\
\text { DE ENSINO }\end{array}$ \\
\hline 2012 & $\begin{array}{c}\text { Constituição química e avaliação das atividades antibacteriana e } \\
\text { esquistossomicida in vitro dos óleos essenciais de Ageratum conzyzoides } \\
\text { l. (Asteraceae) e de Tetradenia riparia (Hochst.) Codd. (Lamiaceae) }\end{array}$ & UNIFRAN \\
\hline 2012 & Estudo farmacobotânico de seis espécies de uso medicinal no nordeste brasileiro & UFPB \\
\hline
\end{tabular}

Nota: UFC - Universidade Federal do Ceará; UFMG - Universidade Federal de Minas Gerais; UNESP - Universidade Estadual Paulista Júlio de Mesquita Filho/*Jaboticabal/**Botucatu; UFV -Universidade Federal de Viçosa; UEM - Universidade Estadual de Maringá; UNICAMP -Universidade Estadual de Campinas; USP - Universidade de São Paulo; UFRPE - Universidade Federal Rural de Pernambuco; UFRRJ - Universidade Federal Rural do Rio de Janeiro; UEL - Universidade Estadual de Londrina; UNESC - Universidade do Extremo Sul Catarinense; UFLA - Universidade Federal de Lavras; UFG - Universidade Federal de Goiás; UEFS - Universidade Estadual de Feira de Santana; UFSC - Universidade Federal de Santa Catarina; UFPB - Universidade Federal da Paraíba; UNIFRAN - Universidade de Franca.

Fonte: Coordenação de Aperfeiçoamento de Pessoal de Nível Superior (2018)

A maior parte dos projetos de pós-graduação envolvendo a espécie concentra-se na Região Sudeste do País (57,0\%) (Figura 1) e como pode ser visto no Quadro 1, a maior parte deles refere-se à trabalhos na área agronômica.

Figura 1 - Distribuição das dissertações/teses de Ageratum segundo o estado da instituição de ensino superior no País, para o período anterior a agosto de 2013

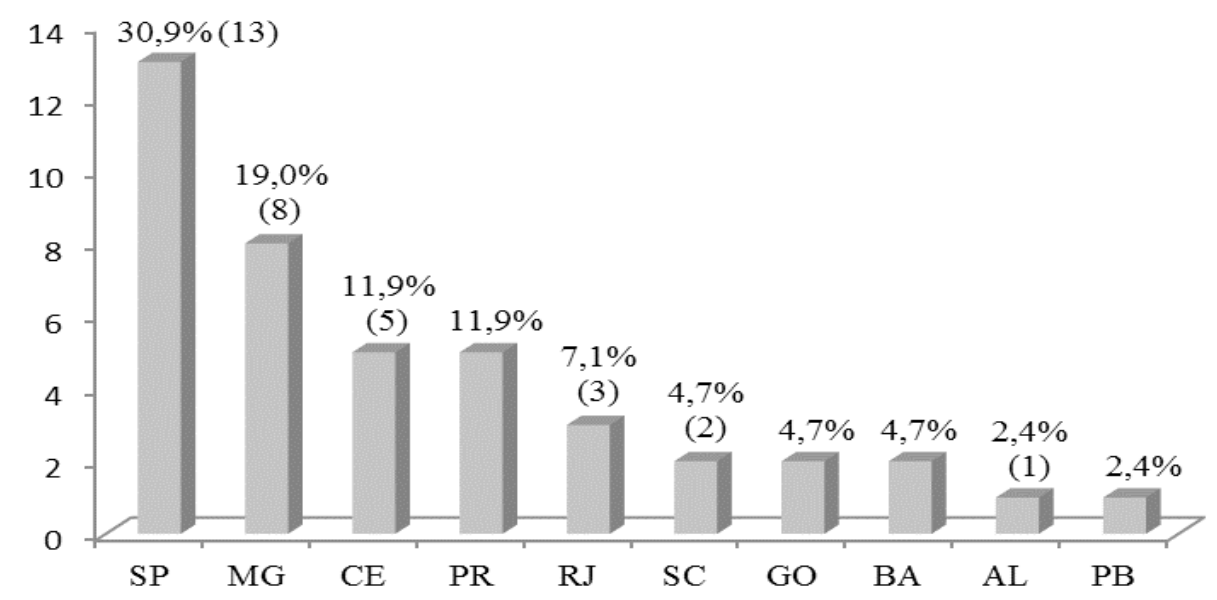

Fonte: Coordenação de Aperfeiçoamento de Pessoal de Nível Superior (2018)

Com relação à prospecção tecnológica, no INPI, apenas um registro foi encontrado (Tabela 3), o qual tratava do uso terapêutico de preparação medicinal, sendo depositada por pessoa física em 2005, no entanto, o pedido encontra-se inviabilizado de proteção patentária por ter sido arquivado definitivamente. A ausência de patentes nacionais não reflete o número de programas de pós-graduação que possuem $A$. conyzoides como objeto de estudo e nem a grande utilização da espécie como recurso terapêutico no País (Quadro 1).

Todas as patentes que constavam na base de dados da OMPI são reportadas na base do $\mathrm{EPO}$, por esse motivo as patentes da OMPI não estão aqui discutidas. A única patente do EPO em que se encontrou a palavra-chave mentrasto, também tinha a palavra Ageratum no resumo, por esse motivo o total de patentes nessa base de patentes são 294. 
Tabela 3 - Termos de busca utilizados nos bancos de patentes e resultados encontrados para o período anterior a janeiro de 2015

\begin{tabular}{|cccc|}
\hline Palavra-chave & INPI & WIPO & EPO \\
\hline Ageratum & 0 & 235 & $294^{*}$ \\
mentrasto & 1 & 0 & 1 \\
\hline
\end{tabular}

Nota: *Na busca apareceram 296, no entanto dois não tinham nem título e nem resumo.

Fonte: Elaborada pelos autores deste artigo, a partir de Instituto Nacional da Propriedade Industrial, Organização Mundial de Propriedade Intelectual e European Patent Office (2018)

Analisando a distribuição das patentes por ano de depósito é possível verificar que o maior número ocorreu no ano de 2008 e 2013, com 49 e 45 solicitações na EPO, respectivamente (Figura 2), sendo a China o país detentor de 90\% (265) das patentes (Tabela 4).

Figura 2 - Evolução anual de patentes referentes à Ageratum depositadas na EPO

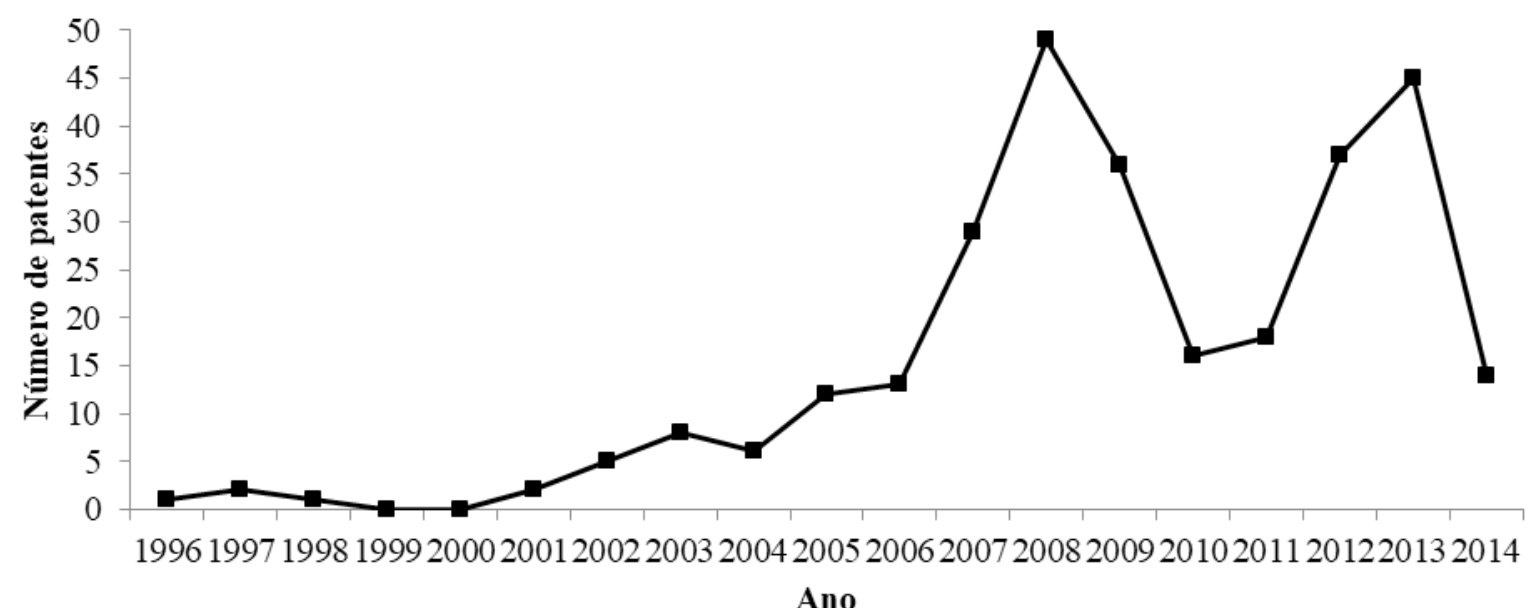

Fonte: European Patent Office (2018)

Com relação à titularidade das patentes, as pessoas jurídicas, empresas ou instituições de pesquisa são detentoras de 180 (61,22\%) dos depósitos de patentes, enquanto pessoas físicas detêm 111 (37,76\%) dos depósitos e apenas 3 (1,02\%) depósitos pertencem à pessoa física e jurídica em parceria.

Tabela 4 - Países depositantes das patentes referentes à Ageratum no banco de dados da EPO

\begin{tabular}{ccc} 
País depositante & N. & $\mathbf{\%}$ \\
China & 265 & 90,14 \\
Países Baixos & 12 & 4,08 \\
Japão & 9 & 3,06 \\
Estados Unidos & 3 & 1,02 \\
Suíça & 3 & 1,02 \\
Coreia & 1 & 0,34 \\
India & 1 & 0,34 \\
Total & $\mathbf{2 9 4}$ & $\mathbf{1 0 0}$ \\
\hline
\end{tabular}

Fonte: European Patent Office (2018) 
Das 294 patentes da EPO, apenas 25 especificavam o representante do gênero no título e/ ou resumo do documento (Tabela 5).

Tabela 5 - Número de patentes para o termo de busca Ageratum no banco de dados da EPO

\begin{tabular}{ccc} 
EsPÉcIE & N. & $\mathbf{\%}$ \\
Ageratum sp. & 269 & 91,50 \\
A. conyzoides & 22 & 7,48 \\
A. houstonianum & 1 & 0,34 \\
A. conyzoides e A. houstonianum & 2 & 0,68 \\
Total & $\mathbf{2 9 4}$ & $\mathbf{1 0 0}$ \\
\hline
\end{tabular}

Fonte: European Patent Office (2018)

Os 294 depósitos de patentes para Ageratum encontram-se alocados em 45 subclasses da CIP e a maioria dos depósitos são pertencentes à subclasse A61K, seguidos da subclasse A61P e A23L (Figura 2), sendo que as subclasses que só tiveram uma indicação de patente (A61F, A61J, A21D, A24F, A43B, A01M, A23C, A24D, A41B, A23B, B09C, C11B, C12Q, C12J, C12M, C12P, C12N, C07C, C07H, C07K, C04B, D21H e D06M) não estão representadas na figura. Os depósitos de patentes para o gênero prevalecem nas classes referentes às ciências médicas (A61), alimentos (A23) e agricultura (A01) (Figura 3).

Figura 3 - Distribuição por subclasse da CIP dos depósitos de patentes para Ageratum encontrados na EPO

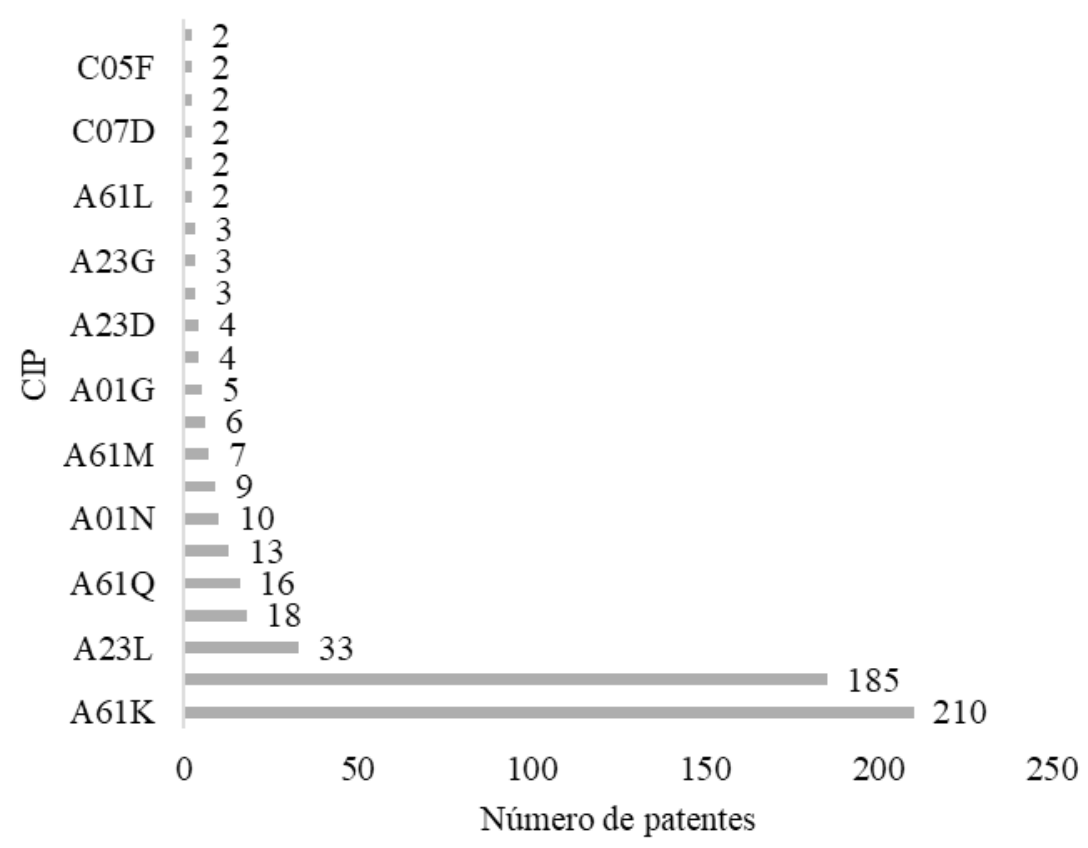

Fonte: European Patent Office (2018)

Os 25 depósitos de patentes para A. conyzoides e/ou A. houstonianum encontram-se alocados em 13 subclasses da CIP, nas áreas de necessidades humanas (A), operações de processamento (B) e química e metalurgia (C) (Quadro 2), sendo a maioria dos depósitos indicados 
na subclasse A61K, seguidos da subclasse A61P e A61Q (Figura 4). A CIP serve de base para investigar e avaliar o estado da técnica em determinado campo da tecnologia (INPI, 2017), no caso de A. conyzoides e/ou A. houstonianum, todas as patentes na classe A61 têm interface com a área farmacêutica (Quadro 2).

Com relação ao depositante das 25 patentes que constam para as espécies $A$. conyzoides e/ ou $A$. houstonianum, $60 \%$ pertencem a pessoas jurídicas e o restante a pessoas físicas. A maior parte dos detentores, como pessoa jurídica, são instituições de pesquisa $(66,7 \%)$, seguida de empresas $(20 \%)$ e em menor número de titularidade, empresas em parceria com instituições de pesquisa (13,3\%). Com relação ao status legal das patentes de $A$. conyzoides, 41,7\% delas estão em avaliação, 25,0\% foram concedidas, 20,8\% indeferidas e 12,5\% já estão em domínio público, ou seja, já se atingiram dez anos de uso da patente (Quadro 3).

Figura 4 - Distribuição por subclasse da CIP dos depósitos de patentes para A. conyzoides e/ou A. houtonianum encontrados na EPO

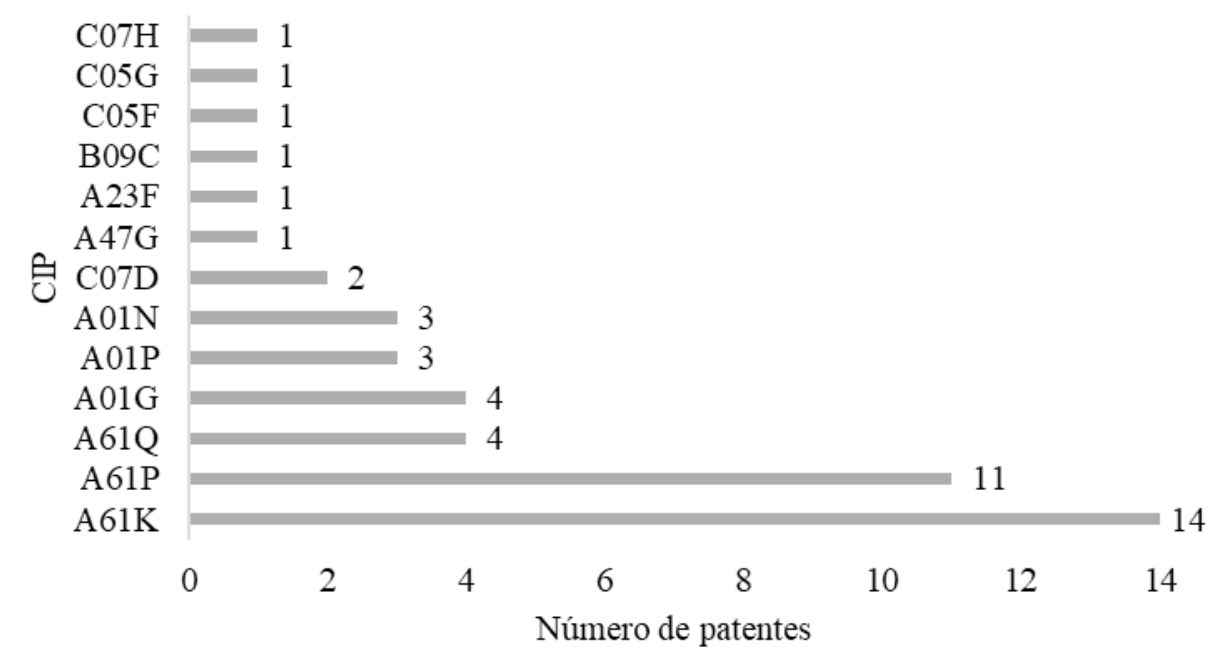

Fonte: European Patent Office (2018)

Quadro 2 - Subclasses das patentes encontradas para A. conyzoides e/ou A. houstonianum no banco de dados da EPO, segundo a CIP

\begin{tabular}{|c|c|}
\hline CIP & Patentes \\
\hline A61K & Preparações para finalidades médicas, odontológicas ou higiênicas \\
\hline A61P & Atividade terapêutica específica de compostos químicos ou preparações medicinais \\
\hline A61Q & Uso específico de cosméticos ou preparações similares para higiene pessoal \\
\hline $\mathrm{A} 01 \mathrm{G}$ & Horticultura; cultivo de vegetais, flores, arroz, frutas, vinhas, lúpulos ou algas; silvicultura; irrigação \\
\hline $\mathrm{A} 01 \mathrm{~N}$ & $\begin{array}{l}\text { Conservação de corpos de seres humanos ou animais ou plantas ou partes } \\
\text { dos mesmos; Biocidas, p. ex., como desinfetantes, pesticidas ou herbicidas; } \\
\text { repelentes ou atrativos de pestes; reguladores do crescimento de plantas }\end{array}$ \\
\hline A01P & $\begin{array}{c}\text { Atividade de compostos químicos ou preparações biocidas, repelentes ou } \\
\text { atrativos de pestes ou reguladores do crescimento de plantas }\end{array}$ \\
\hline A47G & Equipamento para casa ou mesa \\
\hline A23F & Café, chás, seus substitutos; manufatura, preparo ou infusão dos mesmos \\
\hline
\end{tabular}




\begin{tabular}{|c|c|}
\hline CIP & PATENTES \\
\hline B09C & $\begin{array}{r}\text { Recuperação de solo contaminado } \\
\text { C05G }\end{array}$ \\
$\begin{array}{c}\text { Misturas de fertilizantes pertencendo individualmente a diversas subclasses da classe C05; misturas de } \\
\text { um., pesticidas, condicionadores do solo, agentes umectantes; fertilizantes caracterizados por sua forma. }\end{array}$ \\
\hline C05F & $\begin{array}{r}\text { Fertilizantes orgânicos não abrangidos pelas subclasses C05B, C05C, p. } \\
\text { ex. fertilizantes resultantes do tratamento de lixo ou refugos }\end{array}$ \\
\hline C07D & Compostos heterocíclicos \\
\hline C07H & Açúcares seus derivados; nucleosídeos; nucleotídeos; ácidos nucleicos \\
\hline
\end{tabular}

Fonte: European Patent Office (2018)

As patentes referentes às preparações à base de A. conyzoides reivindicam o uso para tratamento de hemorroidas (CN1720944(A)), doença inflamatória crônica pélvica (CN1733078(A)), clareamento da pele (JP2005008610(A)), tônico capilar (JPH09295919(A); JP2000044439(A)) e inibição da tirosinase melhorando a pigmentação da pele (JPH10265366(A)). Enquanto no levantamento por artigos científicos, somente um ensaio clínico foi localizado, as patentes relatam outros estudos clínicos comprovando o uso eficaz e seguro do preparado medicinal de A. conzyoides na Medicina Tradicional Chinesa para o tratamento da pneumonia (CN103393920(A)), dermatite seborreica (CN103536763(A)) e artrite reumatoide (CN103656216(A)). Esta última indicação terapêutica é a que vem sendo explorada nas FV do País, e ainda assim nenhum depósito de patente foi encontrado.

Quadro 3 - Patentes depositadas no EPO referentes à A. conyzoides e/ou A. hostonianum

\begin{tabular}{|c|c|c|c|c|c|}
\hline Ano & Título & CIP & Status & TitulaR & País DEPOSITANTE \\
\hline 1975 & Anti-juvenile hormones & C07D & - & PJ & Estados Unidos \\
\hline 1996 & Hair restoring agent & A61K; A61Q & I & PJ & Japão \\
\hline 1997 & Tyrosinase inhibitor & A61K; A61Q & I & PJ & Japão \\
\hline 1998 & $\begin{array}{l}\text { Composition for scalp } \\
\text { and hair treatment }\end{array}$ & A61K; A61P; A61Q & I & PJ & Japão \\
\hline 2003 & Skin external preparation & A61K; A61Q & I & $\mathrm{PF}$ & Japão \\
\hline 2005 & $\begin{array}{l}\text { Medicine for treating piles } \\
\text { and its preparing process }\end{array}$ & A61K; A61P & A & $\mathrm{PF}$ & China \\
\hline 2005 & $\begin{array}{l}\text { Heat clearing tablet of tropic } \\
\text { ageratum and preparation } \\
\text { process thereof }\end{array}$ & A61K; A61P & A & $\mathrm{PF}$ & China \\
\hline 2006 & $\begin{array}{l}\text { Shenghong heat-clearing granule } \\
\text { and its preparation method }\end{array}$ & A61K; A61P & $\mathrm{C}$ & PJ* & China \\
\hline 2007 & Health pillow with Chinese medicine & A47G; A61K; A61P & A & $\mathrm{PF}$ & China \\
\hline 2009 & $\begin{array}{l}\text { Bacteriostatic component extracted } \\
\text { from Ageratum conyzoides and } \\
\text { extraction method thereof }\end{array}$ & A61K; A61P; C07H & A & PJ & China \\
\hline 2010 & $\begin{array}{l}\text { Peach garden using landscape } \\
\text { plants to control peach tree pests }\end{array}$ & A01G & C & PJ & China \\
\hline
\end{tabular}




\begin{tabular}{|c|c|c|c|c|c|}
\hline Ano & Título & CIP & Status & Titular & País DEPositante \\
\hline 2010 & $\begin{array}{l}\text { Method for remedying } \\
\text { organochlorine pesticide- } \\
\text { induced soil pollution by using } \\
\text { Ageratum conyzoides L }\end{array}$ & B09C & I & PJ & China \\
\hline 2011 & $\begin{array}{l}\text { Method for controlling exotic } \\
\text { Ageratum conyzoides }\end{array}$ & A01G; C05G & C & PJ & China \\
\hline 2011 & $\begin{array}{l}\text { Method of using native plants } \\
\text { to control growth of exotic } \\
\text { Ageratum conyzoides }\end{array}$ & A01G; C05F & $\mathrm{C}$ & PJ & China \\
\hline 2012 & $\begin{array}{l}\text { Application of alien invasive } \\
\text { plant Ageratum conyzoides L. in } \\
\text { control of golden apple snail }\end{array}$ & A01N; A01P & A & PJ & China \\
\hline 2012 & $\begin{array}{l}\text { Vulnerary hemostatic and } \\
\text { preparation technology thereof }\end{array}$ & A61K; A61P & C & $\mathrm{PF}$ & China \\
\hline 2012 & $\begin{array}{l}\text { Weedicide composition containing } \\
\text { glyphosate, fluroxypyr-meptyl } \\
\text { and carfentrazone-ethyl }\end{array}$ & A01N; A01P & A & PJ & China \\
\hline 2013 & $\begin{array}{l}\text { Spleen-tonifying heart-nourishing } \\
\text { health-care tea cream and } \\
\text { preparation method thereof }\end{array}$ & A23F & C & PJ & China \\
\hline 2013 & $\begin{array}{l}\text { Traditional Chinese medicine for } \\
\text { treating rheumatoid arthritis }\end{array}$ & A61K; A61P & A & $\mathrm{PF}$ & China \\
\hline 2013 & $\begin{array}{l}\text { Traditional Chinese medicine } \\
\text { powder for treating pneumonia }\end{array}$ & A61K; A61P & $\mathrm{P}$ & $\mathrm{PF}$ & China \\
\hline 2013 & $\begin{array}{l}\text { Traditional Chinese medicine for } \\
\text { treating seborrheic dermatitis }\end{array}$ & A61K; A61P & $\mathrm{P}$ & $\mathrm{PF}$ & China \\
\hline 2013 & $\begin{array}{l}\text { Method for extracting purified corymb } \\
\text { Ageratum conyzoides element } C\end{array}$ & C07D & $P$ & PJ & China \\
\hline 2013 & $\begin{array}{l}\text { Herbicide composition containing } \\
\text { glufosinate ammonium, glyphosate } \\
\text { and carfentrazone-ethyl }\end{array}$ & A01N; A01P & A & PJ & China \\
\hline 2014 & $\begin{array}{l}\text { Qi-tonifying and sedative } \\
\text { granules for acute heart failure } \\
\text { and preparation method }\end{array}$ & A61K; A61P & A & $\mathrm{PF}$ & China \\
\hline 2014 & $\begin{array}{l}\text { Method for blending and } \\
\text { interplanting plants in organic orchard }\end{array}$ & $\mathrm{A} 01 \mathrm{G}$ & A & PJ & China \\
\hline
\end{tabular}

Nota: CIP - Classificação Internacional de Patentes; PF - pessoa física; PJ - pessoa jurídica; * O depositante da patente consta como PF, porém em 2012 ocorreu transferência de titularidade para pessoa jurídica; $\mathrm{C}$ - concedida; $\mathrm{A}$ - em avaliação; $\mathrm{P}$ - em domínio público; I - indeferido.

Fonte: European Patent Office (2018)

Segundo Barros (2014), das patentes referentes às subclasses A61K e A61P em todo o mundo, apenas $2 \%$ foram depositadas no Brasil, sendo que a maioria desses depositantes são estrangeiros. O que evidencia a discrepância entre o que é produzido pelas instituições de ensino e pesquisa no País e o que é protegido. Esse paradoxo torna-se mais expressivo ao saber que o Brasil é detentor de 20\% da biodiversidade no mundo (MINISTÉRIO DO MEIO AMBIENTE, 2018), assim tal condição deveria propiciar o lançamento de produtos inovadores a partir da flora nacional, mas o faturamento do setor de fitoterápicos no País em 2014 foi de apenas 2\% do faturamento total da indústria farmacêutica. Entretanto, o mercado é promissor, pois há 
uma tendência mundial na sociedade moderna pela busca de terapêuticas menos agressivas, propiciando um aumento gradual do mercado de fitoterápicos o qual, no Brasil, cresceu $6 \%$ em 2014 e 8\% em 2015 (VALÉCIO, 2016).

A exploração de bioativos não se restringe à indústria farmacêutica, pois a busca por tecnologias agrícolas mais verdes é crescente. Estima-se que o mercado de biopesticidas atingirá o valor de US\$ 4,5 bilhões em 2023, correspondendo a aproximadamente $7 \%$ do mercado de pesticidas (AGROLINK, 2014). O expressivo crescimento do mercado de biopesticidas é explicado pela preocupação com as mudanças climáticas e os impactos ambientais da agricultura, além do fato do defensivo biológico, quando utilizado em alternância com os produtos químicos, permitir um prolongamento da vida útil desses últimos. No Brasil, as indústrias de defensivos agrícolas biológicos registraram 20 novos produtos em 2015, um crescimento de mais de $135 \%$ sobre a média dos últimos seis anos (ORGANICSNET, 2015). No entanto, nenhuma patente nacional pertencente à classe A01 (agricultura), envolvendo Ageratum, foi encontrada na busca realizada, apesar dos diversos estudos nos programas de pós-graduação do País com as espécies do gênero (Quadro 1).

Dessa forma, diante das publicações científicas e grupos de pesquisa no País investigando os representantes do gênero, fica evidente o potencial de crescimento do mercado brasileiro em fitoterápicos e defensivos agrícolas a partir dessas espécies, não se refletindo no número de patentes nacionais, visto a ausência de registro. Para auxiliar a alavancar esse setor deve-se destacar a importância das políticas públicas na promoção do patenteamento das invenções a partir da biodiversidade brasileira. Além disso, deve ser incentivada a utilização da patente em domínio público como fonte de informação tecnológica e vantagem competitiva para a produção de produtos.

\section{Considerações Finais}

Neste estudo, foi possível obter o mapeamento de patentes e publicações científicas relacionadas ao gênero Ageratum, notando-se um maior número de artigos científicos publicados (761) do que depósitos de patentes (294), ambos com maior representatividade para a espécie A. conyzoides. De acordo com o estudo prospectivo, pôde-se verificar que a maioria das patentes depositadas foi no ano de 2008 e 2013, sendo a China o país que se destaca com maior número de patentes. Da prospecção tecnológica realizada, a maior parte corresponde a patentes classificadas como A61K (preparações para finalidades médicas, odontológicas ou higiênicas) e A61P (atividade terapêutica específica de compostos químicos ou preparações medicinais). Apesar das 37 teses/dissertações desenvolvidas nos programas de pós-graduação do País envolvendo a espécie $A$. conyzoides, nenhum registro de patente nacional, em avaliação ou concedido, foi encontrado nos bancos de patentes pesquisados. Com isto, vê-se a necessidade de um maior incentivo para que as pesquisas realizadas no País gerem propriedade intelectual $e$ assim intensifiquem as investigações em busca de um melhor aproveitamento comercial da biodiversidade brasileira, principalmente no que tange ao potencial medicinal, agronômico $e$ 
alimentar para os representantes do gênero, uma vez que A. conyzoides tem sido utilizada para esses fins em diversas culturas no mundo, especialmente no Brasil.

\section{Referências}

AGROLINK. Mercado dos biopesticidas deve crescer US\$ 4,5 bilhões até 2023. 2014.

Disponível em: < https:/www.agrolink.com.br/noticias/mercado-dos biopesticidas-deve-crescer-us--45-bilhoes-ate-2023_191603.html>. Acesso em: 13 jun. 2017.

BARROS, W. B. G. Patente como fonte de informação tecnológica: utilização de documentos de patente em domínio público. In: SIMPÓSIO INTERNACIONAL DE GESTÃO DE PROJETOS E II SIMPÓSIO INTERNACIONAL DE INOVAÇÃO E SUSTENTABILIDADE, III., São Paulo, nov. 2014. Anais... São Paulo, 2014. p. 1-10. Disponível em: <http://docplayer.com.br/1557320-Patentecomo-fonte-de-informacao-tecnologica-utilizacao-de-documentos-de-patente-em-dominio-publico. html>. Acesso em: 31 maio 2016.

CENTRO LATINO-AMERICANO E DO CARIBE DE INFORMAÇÃO EM CIÊNCIAS DA SAÚDE (BIREME). Base de dados on-line. [2018]. Disponivel em: <https://bvsalud.org///> . Acesso em: 10 jan. 2018.

BOSI, C. F. et al. Pyrrolizidine alkaloids in medicinal tea of Ageratum conyzoides. Revista Brasileira de Farmacognosia, Curitiba, v. 23, n. 3, p. 425-432, 2013.

BRASIL. Ministério da Saúde, Secretaria de Ciência, Tecnologia e Insumos Estratégicos, Departamento de Assistência Farmacêutica. Tecnologia e insumos estratégicos. Departamento de Assistência Farmacêutica. A fitoterapia no SUS e o Programa de Pesquisa de Plantas Medicinais da Central de Medicamentos. Brasília, DF: Ministério da Saúde, 2006. Disponível em: $<$ http://bvsms.saude.gov.br/bvs/publicacoes/fitoterapia_no_sus.pdf > . Acesso em: 14 out. 2018.

BUENO, D. Caminhos para proteção intelectual. Revista FAPESP, São Paulo, ed. 217, p. 95-96, mar. 2014.

CARVALHO, A. C. B. Plantas medicinais e fitoterápicos: regulamentação sanitária e proposta de modelo de monografia para espécies vegetais oficializadas no Brasil. 2011. 318 f. Tese (Doutorado em Ciências da Saúde) - Programa de Pós-Graduação em Ciências da Saúde, Universidade de Brasília, Brasília, DF, 2011.

COORDENAÇÃO DE APERFEIÇOAMENTO DE PESSOAL DE NÍVEL SUPERIOR (Capes). Base de dados on-line. [2018]. <http://www.periodicos.capes.gov.br/>. Acesso em: 10 jan. 2018.

COSTA, M. A. et al. Plantas \& Saúde: guia introdutório à fitoterapia. Brasília, DF: Governo do Distrito Federal, 1992.

DEL-VECHIO-VIEIRA, G. et al. Caracterização morfoanatômica de Ageratum fastigiatum (Asteraceae). Revista Brasileira de Farmacognosia, Curitiba, v. 18, Supl., p. 769-776, dez. 2008.

GRIFFIN, M. R. High-dose non-steroidal anti-inflammatories: painful choices. The Lancet, [S.l.], v. 382, n. 9.894, p. 746-748, ago. 2013.

CENTRO DE DISSEMINAÇÃO DA INFORMAÇÃO TECNOLÓGICA (CEDIN). Introdução à Classificação Internacional de Patentes. 2017. Disponível em: <file://C:/Users/User/Downloads/ TUTORIALDECLASSIFICACAOIPCatualizacao311122017Anexo1.pdf > . Acesso em: 14 out. 2018. 
EUROPEAN PATENT OFFICE (EPO). Base de dados on-line. [2018]. Disponível em: < https:// worldwide.espacenet.com/advancedSearch?locale=en_EP>. Acesso em: 10 jan. 2018.

INSTITUTO NACIONAL DA PROPRIEDADE INDUSTRIAL (INPI). Base de dados on-line. [2018]. Disponível em: <https:/gru.inpi.gov.br/pePI/jsp/patentes/PatenteSearchAvancado.jsp >. Acesso em: 10 jan. 2018.

JAWAID, T.; SHUKLA, D.; VERMA, J. Anti-inflammatory activity of the plants used in traditional medicines. International Journal of Biomedical Research, [S.l.], v. 2, n. 4, p. 252-263, 2011.

KAMBOJ, A.; SALUJA, A. K. Ageratum conyzoides L.: a review on its phytochemical and pharmacological profile. International Journal of Green Pharmacy, [S.l.], v. 2, n. 2, p. 59-68, 2008.

KAUR, S. et al. Ageratum conyzoides: An alien invasive weed in India. In: BHATT, J.R. et al. Invasive alien plants: an ecological appraisal for the indian subcontinent. p. 57-76, 2012. Disponível em: <https://books.google.com.br/books/about/Invasive_Alien_Plants_An_Ecological_Appr. html?id=1YDiD0JlUGoC\&redir_esc $=y>$. Acesso em: $1 \overline{4}$ out. 2018.

KONG, C. et al. Allelopathic plants. XV. Ageratum conyzoides L. Allelopathy Journal, [S.l.], v. 14, n. 1, p. 1-12, 2004.

LILACS. Base de dados on-line. [2018]. Disponível em: <https://www.ncbi.nlm.nih.gov/ pubmed/>. Acesso em: 10 jan. 2018.

MARTINELLI, G.; MORAES, M. A. (Org.). Livro vermelho da flora do Brasil. 1. ed. Rio de Janeiro: Andrea Jakobsson/Instituto de Pesquisas Jardim Botânico do Rio de Janeiro, 2013.

MEDLINE. Base de dados on-line. [2018]. Disponível em: <https://www.ncbi.nlm.nih.gov/ pubmed//>. Acesso em: 10 jan. 2018.

MINISTÉRIO DO MEIO AMBIENTE (MMA). Biodiversidade brasileira. 2018. Disponível em: <http://www.mma.gov.br/biodiversidade/biodiversidade-brasileira >. Acesso em: 14 out. 2018

NAKAJIMA, J. N. Ageratum in lista de espécies da flora do Brasil. Jardim Botânico do Rio de Janeiro. 2016. Disponível em: <http://floradobrasil.jbrj.gov.br/jabot/floradobrasil/FB15932>. Acesso em: 18 jan. 2016.

OKUNADE, A. L. Ageratum conyzoides L. (Asteraceae). Fitoterapia, v. 73, n. 1, p. 1-16, fev. 2002.

ORGANICSNET. Mercado de defensivo biológico pode crescer até $\mathbf{2 0 \%}$ ao ano no Brasil. 2015. Disponível em: <http://www.organicsnet.com.br/2015/12/mercado-de-defensivo-biologicopode-crescer-ate-20-ao-ano-no-brasil-2/>. Acesso em: 13 jun. 2017.

ORGANIZAÇÃO MUNDIAL DE PROPRIEDADE INTELECTUAL (WIPO). Base de dados on-line. [2018]. Disponível em: <https://patentscope.wipo.int/search/pt/advancedSearch.jsf > . Acesso em: 10 jan. 2018.

SCIENCE DIRECT. Base de dados on-line. [2018]. Disponível em: <https://www.ncbi.nlm.nih.gov/ pubmed/>. Acesso em: 10 jan. 2018.

SCIENTIFIC ELECTRONIC LIBRARY ONLINE (SciELO). Base de dados on-line. [2018].

Disponível em: <http://www.scielo.org > . Acesso em: 10 jan. 2018.

SCIFINDER. Base de dados on-line. [2018]. Disponível em: <https://scifinder.cas.org>. Acesso em: 10 jan. 2018. 
SCOPUS. Base de dados on-line. [2018]. Disponível em: <https://www.scopus.com/home.uri>. Acesso em: 10 jan. 2018.

SERAFINI, M. R. et al. Mapeamento de tecnologias patenteáveis com o uso da hecogenina. Revista Geintec, [S.l.], v. 2, n. 5, p. 427-435, out./nov./dez. 2012.

SILVA, M. I. G. et al. Utilização de fitoterápicos nas unidades básicas de atenção à saúde da família no município de Maracanaú (CE). Revista Brasileira de Farmacognosia, Curitiba, v. 16, n. 4, p. 455-462, out./dez. 2006.

VALÉCIO, M. Fitoterápicos ganham espaço. Guia da Farmácia, [S.1.], Suplemento Especial Fito, v. 23, n. 281, p. 14-17, abr. 2016. Disponível em: < http://www.guiadafarmacia.com.br/suplementosespeciais/fito/fito-2016/10792-fitoterapicos-ganham-credibilidade > . Acesso em: 14 jun. 2017.

\section{Sobre os Autores}

\section{Ingrid Estefania Mancia de Gutiérrez}

E-mail: far_gutierrez@yahoo.com.br

Formação: Doutora em Biotecnologia.

Endereço profissional: Av. Transnordestina, s/n., Campus Universitário, Bairro Novo Horizonte - Feira de Santana, BA. CEP: 44036-900.

\section{Daniel da Silva de Jesus}

E-mail: dasilva_jesus@yahoo.com.br

Formação: Doutor em Recursos Genéticos Vegetais.

Endereço profissional: Av. Transnordestina, s/n., Campus Universitário, Bairro Novo Horizonte - Feira de Santana, BA. CEP: 44036-900.

\section{Lenaldo Muniz de Oliveira}

E-mail: lenaldo@uefs.br

Formação: Doutor em Fisiologia Vegetal.

Endereço profissional: Av. Transnordestina, s/n., Campus Universitário, Bairro Novo Horizonte - Feira de Santana, BA. CEP: 44036-900.

\section{Angélica Maria Lucchese}

E-mail: angelica.lucchese@gmail.com

Formação: Doutora em Química Orgânica.

Endereço profissional: Av. Transnordestina, s/n., Campus Universitário, Bairro Novo Horizonte - Feira de Santana, BA. CEP: 44036-900. 